\title{
APLIKASI DISTRIBUSI DERET PANGKAT PADA BEBERAPA JENIS DISTRIBUSI KHUSUS
}

Power Series Distribution Applications in Several Types of Special Distributions

\author{
MOZART WINSTON TALAKUA \\ Staf Jurusan Matematika FMIPA UNPATTI \\ Jl. Ir. M. Putuhena, Kampus Unpatti, Poka-Ambon \\ e-mail: ocat_08@yahoo.com
}

\begin{abstract}
This research about the definition and theorem of power series distribution according to its analitycal characteristics. Then it discusse about the application to another distribution, and discusse about the relation between it, but it especially discusse about the application of power series distribution on some other special distribution, such as the negative logarithm distribution, the divided logarithm distribution, and invers sinus distribution.
\end{abstract}

Keywords: Distribution, power series, negative logarithm, divided logarithm, invers sinus

\section{PENDAHULUAN}

Peluang (probabilitas) berawal dari sebuah perjudian yang dilakukan oleh matematikawan dan fisikawan Italy, yaitu Girolamo Cardan (1501 - 1576) yang ditulis dalam bukunya yang berjudul Liber de Ludo Aleae (Book On Games Of Changes) pada tahun 1565 yang banyak membahas tentang masalah perjudian. Peluang kemudian dibahas oleh para ahli hingga sekarang.

Distribusi normal adalah karya dari Abraham de Moivre yang diperkenalkan pertama kali pada tahun 1737, kemudian ditulis ulang pada tahun 1738 dengan judul The Doctrime of Chances yang membahas tentang pendekatan distribusi binomial untuk $n$ yang besar, kemudian dilanjutkan oleh Laplace dalam bukunya yang berjudul Analytical Theory Of Probability pada tahun 1812, yang sekarang dikenal dengan Teorema De MoivreLaplace. Distribusi kemudian dibahas hingga sekarang, yang dijelaskan oleh Peneliti dalam mengidentifikasi karakteristik parameter untuk model distribusi deret pangkat.

Dalam penelitian ini akan dikaji tentang momen, fungsi pembangkit momen, aplikasi pada beberapa distribusi khusus seperti distribusi deret negatif logaritma, distribusi deret log pembagian, distribusi deret invers sinus (Arcsinus) dari distribusi deret pangkat, serta sifatsifat analitik meliputi Ekepektasi dan variansi.

\section{TINJAUAN PUSTAKA}

Dalam ilmu statistika matematika, teori peluang (probability theory) merupakan dasar dan pengantar untuk penyusunan statistika lebih jauh, dimana dipakai pada penentuan selang untuk distribusi peluang yang terbagi atas distribusi peluang diskrit dan distribusi peluang kontinu (Bain, 1991).

Sebelum membahas lebih jauh tentang fungsi peluang deret pangkat, maka terlebih dahulu diperkenalkan apa yang dinamakan fungsi peluang itu sendiri. Misalkan $\Omega$ suatu himpunan (disebut ruang sampel), dan titik $\omega \in \Omega$ disebut kejadian dasar (unsur). Kemudian misalkan $P(A)$ adalah fungsi peluang untuk setiap kejadian $A \subseteq \Omega$ yang berkaitan dengannya suatu bilangan $P(A)$ sedemikian sehingga $P(A) \geq 0$ dan $P(\Omega)=1$ (Kreyszig, 1993).

Model fungsi peluang lain yang perlu diperkenalkan dan dipelajari dalam statistika adalah deret pangkat:

$$
\sum_{n=0}^{\infty} a_{n} X^{n}=a_{0} X^{0}+a_{1} X^{1}+a_{2} X^{2}+\ldots
$$

yang hanya ditemukan pada analisis metode dan bagian penentuan fungsi peluang deret pangkat (Dudewicz, 1995). 


\section{Deret Pangkat.}

\section{Definisi 1}

Diketahui $z_{1}, z_{2}, \ldots, z_{m}$ barisan bilangan riil, didefinisikan barisan jumlah parsial yaitu:

$$
\begin{aligned}
S_{1} & =z_{1} \\
S_{2} & =z_{1}+z_{2} \\
& \vdots \\
S_{m} & =z_{1}+z_{2}+\ldots+z_{m}
\end{aligned}
$$

Jumlah parsial deret ini boleh tak hingga atau biasa disebut deret dan ditulis :

$$
\sum_{m=1}^{\infty} z_{m}=z_{1}+z_{2}+\ldots
$$

dalam hal ini $z_{m}$ disebut suku dari deret tersebut.

\section{Definisi 2}

Deret pangkat dari $\left(z-z_{0}\right)$ berhubungan dengan,

$$
\sum_{n=0}^{\infty} a_{n}\left(z-z_{0}\right)^{n}=a_{0}+a_{1}\left(z-z_{0}\right)+a_{2}\left(z-z_{0}\right)^{2}+\ldots,
$$

$\left|z-z_{0}\right|<R$, dengan $z$ adalah peubah dan $a_{0}, a_{1}, a_{2}, \ldots$ adalah konstanta yang di namakan koefisien dari deret tersebut, $z_{0}$ adalah konstanta pusat deret. Jika $z_{0}=0$, maka didapat $\sum_{n=0}^{\infty} a_{n} z^{n}=a_{0}+a_{1} z+a_{2} z^{2}+\ldots$

\section{Penurunan Deret Pangkat}

Pada deret pangkat diperbolehkan menurunkan suku demi suku deret tersebut, sebagaimana Definisi 2. Penurunan ini dinamakan turunan pertama dari deret pangkat pada Definisi 2 yaitu ;

$$
\sum_{n=0}^{\infty} n a_{n} z^{n-1}=a_{1}+2 a_{2} z+3 a_{3} z^{2}+\ldots,|z|<R
$$

\section{Teorema 1}

Suatu deret pangkat dengan jari-jari kekonvergenan $R$ yang tidak nol menggunakan suatu fungsi didalam daerah interior universal kekonvergenannya. Turunan fungsi ini diperoleh dengan mendiferensialkan deret asalnya suku demi suku. Semua deret yang diperoleh dengan cara ini menggunakan jari-jari kekonvergenan yang sama dengan deret asalnya.

$$
\frac{d\left(\sum_{n=0}^{\infty} a_{n} z^{n}\right)}{d z}=\sum_{n=0}^{\infty} \frac{d\left(a_{n} z^{n}\right)}{d z},|z|<R
$$

\section{Bukti}

$$
\begin{aligned}
\frac{d\left(\sum_{n=0}^{\infty} a_{n} z^{n}\right)}{d z} & =\frac{d\left(a_{0} z^{0}+a_{1} z^{1}+a_{2} z^{2}+\ldots\right)}{d z} \\
& =\frac{d}{d z}\left(a_{0} z^{0}\right)+\frac{d}{d z}\left(a_{1} z^{1}\right)+\frac{d}{d z}\left(a_{2} z^{2}\right)+\ldots
\end{aligned}
$$

$\frac{d\left(\sum_{n=0}^{\infty} a_{n} z^{n}\right)}{d z}=\sum_{n=0}^{\infty} \frac{d}{d z}\left(a_{n} z^{n}\right)$

$\sum_{n=0}^{\infty} \frac{d}{d z}\left(a_{n} z^{n}\right)=\frac{d}{d z}\left(a_{0} z^{0}\right)+\frac{d}{d z}\left(a_{1} z^{1}\right)+\frac{d}{d z}\left(a_{2} z^{2}\right)+\ldots$ $=\frac{d}{d z}\left(a_{0} z^{0}+a_{1} z^{1}+a_{2} z^{2}+\ldots\right)$

$\sum_{n=0}^{\infty} \frac{d}{d z}\left(a_{n} z^{n}\right)=\frac{d\left(\sum_{n=0}^{\infty} a_{n} z^{n}\right)}{d z}$

dari (1) dan (2) maka terbukti bahwa

$$
\frac{d\left(\sum_{n=0}^{\infty} a_{n} z^{n}\right)}{d z}=\sum_{n=0}^{\infty} \frac{d\left(a_{n} z^{n}\right)}{d z}
$$

\section{Fungsi Distribusi}

\section{Definisi 3}

Jika himpunan semua kemungkinan nilai peubah acak $x$ adalah himpunan hingga $x_{1}, x_{2}, \ldots, x_{n}$ atau tak hingga $x_{1}, x_{2}, \ldots$ maka $x$, disebut peubah acak deret, fungsi $f(x)=P(X=x), \quad x=x_{1}, x_{2}, x_{3}, \ldots \quad$ yang dianggap peluang untuk setiap himpunan nilai $X$, yang akan disebut fungsi distribusi peluang.

\section{Fungsi Pembangkit Momen}

\section{Definisi 4}

Jika $X$ peubah acak diskrit maka momen ke- $t$

$$
M_{x}(t)=E\left(e^{t x}\right)=\sum_{x} e^{t x} P(X)
$$

disebut fungsi pembangkit momen dari $X$, jika nilai harapannya ada untuk semua nilai $t$ pada interval $-h<t<h$ dan $h>0$.

\section{Teorema 3}

Jika fungsi pembangkit momen $M_{x}(t)$ dari peubah acak $X$ ada untuk $|t|<h$ dan $h>0$, maka $E\left(X^{k}\right)$ ada dan

$$
E\left(X^{k}\right)=\left.\frac{d M_{x}^{k}(t)}{d t^{k}}\right|_{t=0}
$$

\section{Bukti}

Karena $M_{x}(t)$ ada untuk $|t|<h$ maka dapat mengambil turunannya secara beruruturutan dengan menurunkannya di dalam tanda integral secara terurut, jadi ;

$$
\begin{aligned}
M_{x}^{(k)}(t) & =\frac{d^{k}}{d t^{k}} M_{x}(t)=E\left[\frac{\partial}{\partial t} \frac{d^{k-1}}{d t^{k-1}} e^{t X}\right] \\
& =E\left[X^{k} e^{t X}\right]
\end{aligned}
$$

Jika dimasukan $t=0$, diperoleh $M_{x}^{(k)}(0)=E\left(X^{k}\right)$ 


\section{HASIL DAN PEMBAHASAN}

Suatu deret pangkat sesuai Definisi 2 yang seluruhnya terhitung bisa berhingga atau tak berhingga sesuai Definisi 5 yaitu :

$$
f(z)=\sum_{x} a_{x} z^{x}
$$

dengan $f(z)$ hingga (positif) dan terdefinisi, dan selanjutnya berdasarkan Teorema 2 didapatkan suatu fungsi peluang diskrit $P(X)$ yaitu :

$$
P(X=x)=\frac{a_{x} z^{x}}{f(z)}, a_{x}>0, z>0, x=0,1,2, \ldots
$$

dengan demikian $P(X)$ merupakan suatu fungsi peluang distribusi yaitu (Persamaan 4) yang dinamakan distribusi deret pangkat.

\section{Hubungan Antara Momen}

Hubungan antara momen dapat diperoleh dengan menggunakan :

\section{Teorema 4}

Jika $M_{k}^{\prime}$ momen ke- $k$ dan $M_{1}^{\prime}$ momen pertama maka momen ke- $(k+1)$ atau $M_{k+1}^{\prime}=M_{1}^{\prime} M_{k}^{\prime}+z \frac{d M_{k}^{\prime}}{d z}$

\section{Bukti}

Perhatikan $M_{k}^{\prime}=\frac{1}{f(z)} \sum_{x=1}^{n} x^{k} a_{x} z^{x}$,

selanjutnya didiferensialkan terhadap $z$

$$
\begin{aligned}
\frac{d M_{k}^{\prime}}{d z} & =\frac{1}{f(z)} \sum_{x=1}^{n} x^{k} a_{x} x z^{x-1}-\frac{f^{\prime}(z)}{f^{2}(z)} \sum_{x=1}^{n} x^{k} a_{x} z^{x} \\
z \frac{d M_{k}^{\prime}}{d z} & =\frac{1}{f(z)} \sum_{x=1}^{n} x^{k} a_{x} x z^{x}-\frac{f^{\prime}(z)}{f^{2}(z)} \sum_{x=1}^{n} x^{k} a_{x} z^{x+1} \\
& =\frac{1}{f(z)} \sum_{x=1}^{n} x^{k+1} a_{x} z^{x}-\frac{f^{\prime}(z)}{f^{2}(z)} \sum_{x=1}^{n} x^{k} a_{x} z^{x} z \\
& =\frac{1}{f(z)} \sum_{x=1}^{n} x^{k+1} a_{x} z^{x}-\frac{f^{\prime}(z)}{f^{2}(z)} \sum_{x=1}^{n} x^{k} a_{x} z^{x} z \\
& =M_{k+1}^{\prime}-\frac{f^{\prime}(z)}{f(z)} M_{k}^{\prime} z \\
M_{k+1}^{\prime} & =z \frac{f^{\prime}(z)}{f(z)} M_{k}^{\prime}+z \frac{d M_{k}^{\prime}}{d z} \\
& =E(X) M_{k}^{\prime}+z \frac{d M_{k}^{\prime}}{d z} \\
M_{k+1}^{\prime}= & M_{1}^{\prime} M_{k}^{\prime}+z \frac{d M_{k}^{\prime}}{d z}
\end{aligned}
$$

\section{Momen Sentral}

Momen sentral distribusi deret pangkat dapat diproses dengan berdasar pada Definisi 4, momen sentral ke- $k$ yaitu $M_{k}=\frac{1}{f(z)} \sum_{x}\left(x-M_{1}^{\prime}\right)^{k} a_{x} z^{x}$ dari persamaan di atas diperoleh momen-momen sebagai berikut :

$M_{0}=1$, sebab $\frac{1}{f(z)} \sum_{x} a_{x}\left(x-M_{1}^{\prime}\right)^{0} z^{x}=1$

$$
\begin{aligned}
M_{1} & =\frac{1}{f(z)} \sum_{x} a_{x}\left(x-M_{1}^{\prime}\right)^{1} z^{x} \\
& =\frac{1}{f(z)} \sum_{x} a_{x} x z^{x}-\frac{1}{f(z)} M_{1}^{\prime} \sum_{x} a_{x} z^{x} \\
& =M_{1}^{\prime}-\frac{1}{f(z)} M_{1}^{\prime} \sum_{x} a_{x} z^{x} \\
& =\frac{1}{f(z)} \sum_{x} a_{x} x z^{x}-\frac{1}{f(z)} M_{1}^{\prime} \sum_{x} a_{x} z^{x} \\
& =M_{1}^{\prime}-\frac{1}{f(z)} M_{1}^{\prime} \sum_{x} a_{x} z^{x}
\end{aligned}
$$$$
M_{1}=M_{1}^{\prime}\left(1-\frac{1}{f(z)} \sum_{x}^{(3.3 .5)} a_{x} z^{x}\right)=0
$$

$$
\begin{aligned}
M_{2} & =\frac{1}{f(z)} \sum_{x} a_{x}\left(x-M_{1}^{\prime}\right)^{2} z^{x} \\
& =\frac{1}{f(z)} \sum_{x} a_{x}\left(x^{2}-2 x M_{1}^{\prime}+M_{1}^{\prime 2}\right)^{2} z^{x} \\
& =\frac{1}{f(z)} \sum_{x} a_{x} x^{2} z^{x}-\frac{2 M_{1}^{\prime}}{f(z)} \sum_{x} a_{x} x z^{x}+\frac{M_{1}^{\prime 2}}{f(z)} \sum_{x} a_{x} z^{x} \\
& =M_{2}^{\prime}-2 M_{1}^{\prime} M_{1}^{\prime}+M_{1}^{\prime 2}
\end{aligned}
$$

$M_{2}=M_{2}^{\prime}-M_{1}^{\prime 2}$, jika disubstitusikan nilai dari $M_{1}^{\prime}$ dan $M_{2}^{\prime}$ diperoleh

$M_{2}=\operatorname{Var}(X)$

$$
=\frac{1}{f^{2}(z)}\left(z^{2} f^{\prime \prime}(z) f(z)+z f^{\prime}(z) f(z)-z^{2} f^{2}(z)\right)
$$

dengan cara yang sama diperoleh

$$
\begin{aligned}
M_{3}= & M_{3}^{\prime}-3 M_{2}^{\prime} M_{1}^{\prime}+2 M_{1}^{\prime 3} \\
M_{3}= & \frac{1}{f^{3}(z)}\left(f ^ { 2 } ( z ) \left[z^{3} f^{\prime \prime \prime}(z)+3 z^{3} f^{\prime}(z) f^{\prime \prime}(z)\right.\right. \\
& \left.\left.+2 f^{\prime}(z)\right]\right)-\frac{1}{f^{2}(z)}\left(3 z^{2} f^{\prime 2}(z)+3 z^{3} f^{\prime}(z) f^{\prime \prime}(z)\right) \\
& +2 \frac{z^{3} f^{\prime 3}(z)}{f^{3}(z)}
\end{aligned}
$$

sehingga diperoleh

$$
M_{k}=E\left(X-M_{1}^{\prime}\right)=\sum_{s=0}^{k}(-1)^{s}\left(\begin{array}{l}
k \\
s
\end{array}\right) M_{1}^{\prime j} M_{k-s}^{\prime}
$$

akibat dari momen sentral diatas didapatkan momen jika dicari momen sentralnya sebagai berikut :

$$
\begin{aligned}
M_{2}^{\prime} & =M_{2}+M_{1}^{\prime 2} \\
M_{3}^{\prime} & =M_{3}+3 M_{1}^{\prime} M_{2}+M_{1}^{\prime 3} \\
& \vdots \\
M_{k}^{\prime} & =E\left(X-M_{1}^{\prime}+M_{1}^{\prime}\right)=\sum_{s=0}^{k}\left(\begin{array}{l}
k \\
s
\end{array}\right) M_{1}^{\prime j} M_{k-s}
\end{aligned}
$$




\section{Hubungan Antara Momen Sentral}

Hubungan antara momen sentral diperoleh dengan menggunakan Teorema 5 di bawah ini.

\section{Teorema 5}

Jika $M_{k}$ momen sentral ke- $k$ dan $M_{k-1}$ momen sentral ke- $(k-1)$, maka momen sentral ke- $(k-1)$ yaitu :

$$
M_{k+1}=z\left(\frac{d M_{k}}{d z}+k \frac{d M_{1}^{\prime}}{d z} M_{k-1}\right) \text {, dengan } k \text { positif }
$$

\section{Bukti}

Dengan menggunakan nilai dari $M_{k}$ (momen sentral ke-

k) yaitu : $M_{k}=\frac{1}{f(z)} \sum_{x}\left(x-M_{1}^{\prime}\right)^{k} a_{x} z^{x}$

selanjutnya didiferensialkan terhadap z maka didapatkan

$$
\begin{aligned}
& \frac{d M_{k}}{d z}=\frac{1}{f(z)} \sum_{x}\left(x-M_{1}^{\prime}\right)^{k} a_{x} x z^{x-1}-\frac{1}{f(z)} k \frac{d M_{1}^{\prime}}{d z} \\
& \sum_{x}\left(x-M_{1}^{\prime}\right)^{k-1}-\frac{f^{\prime}(z)}{f^{2}(z)} \sum_{x}\left(x-M_{1}^{\prime}\right)^{k} a_{x} z^{x} \\
& \frac{z d M_{k}}{d z}=\frac{1}{f(z)} \sum_{x}\left(x-M_{1}^{\prime}\right)^{k} a_{x} x z^{x} \\
& -z \frac{f^{\prime}(z)}{f^{2}(z)} \sum_{x}\left(x-M_{1}^{\prime}\right)^{k} a_{x} z^{x} \\
& -\frac{1}{f(z)} z^{k} \frac{d M_{1}^{\prime}}{d z} \sum_{x}\left(x-M_{1}^{\prime}\right)^{k-1} a_{x} z^{x} \\
& =\frac{1}{f(z)} \sum_{x}\left(x-M_{1}^{\prime}\right)^{k} a_{x} z^{x}\left(x-z \frac{f^{\prime}(z)}{f(z)}\right) \\
& -\frac{1}{f(z)} z k \frac{d M_{1}^{\prime}}{d z} \sum_{x}\left(x-M_{1}^{\prime}\right)^{k-1} a_{x} z^{x} \\
& =\frac{1}{f(z)} \sum_{x}\left(x-M_{1}^{\prime}\right)^{k} a_{x} z^{x}\left(x-M_{1}^{\prime}\right) \\
& -z k \frac{d M_{1}^{\prime}}{d z} \frac{1}{f(z)} \sum_{x}\left(x-M_{1}^{\prime}\right)^{k-1} a_{x} z^{x} \\
& =\frac{1}{f(z)} \sum_{x}\left(x-M_{1}^{\prime}\right)^{k} a_{x} z^{x}\left(x-M_{1}^{\prime}\right) \\
& -z k \frac{d M_{1}^{\prime}}{d z} \frac{1}{f(z)} M_{k-1} \\
& =\frac{1}{f(z)} \sum_{x}\left(x-M_{1}^{\prime}\right)^{k+1} a_{x} z^{x}-z k \frac{d M_{1}^{\prime}}{d z} \frac{1}{f(z)} M_{k-1} \\
& =M_{k+1}-z k \frac{d M_{1}^{\prime}}{d z} \frac{1}{f(z)} M_{k-1}
\end{aligned}
$$$$
M_{k+1}=z k \frac{d M_{1}^{\prime}}{d z} M_{k-1}+\frac{z d M_{k}}{d z}
$$$$
M_{k+1}=z\left(\frac{d M_{k}}{d z}+k \frac{d M_{1}^{\prime}}{d z} M_{k-1}\right)
$$

\section{Akibat 1}

Jika $M_{k}$ momen sentral ke- $k, M_{2}$ momen sentral ke-2 dan $M_{k-1}$ momen sentral ke- $(k-1)$ maka

\section{Bukti}

$$
M_{k+1}=z \frac{d M_{k}}{d z}+k M_{2} M_{k-1}
$$

Dengan $k=1$ dari Teorema 3, diperoleh :

$$
M_{2}=z\left(\frac{d M_{1}}{d z}+\frac{d M_{1}^{\prime}}{d z} M_{0}\right)
$$

karena $M_{1}=0$ dan $M_{0}=1$ maka, diperoleh

$$
M_{2}=z \frac{d M_{1}^{\prime}}{d z}
$$

dengan demikian persamaan pada Teorema 3 dapat ditulis seperti berikut

$$
M_{k+1}=z \frac{d M_{k}}{d z}+k M_{2} M_{k-1}
$$

\section{Momen Faktorial}

Fungsi pembangkit momen faktorial $G_{x}(t)$, dari model distribusi deret pangkat diperoleh dengan menggunakan

$$
G_{x}(t)=E\left(t^{x}\right)=\sum_{x} t^{x} P(X=x)
$$

sehingga didapatkan,

$$
\begin{aligned}
G_{x}(t) & =\frac{1}{f(z)} \sum_{x} a_{x} t^{x} z^{x} \\
& =\frac{1}{f(z)}\left(a_{1} t z+a_{2}(t z)^{2}+a_{3}(t z)^{3}+\ldots\right) \\
& =\frac{t}{f(z)}\left(a_{1} z+a_{2} t z^{2}+a_{3} t^{2} z^{3}+\ldots\right) \\
& =\frac{t}{f(z)} \sum_{x} a_{x} t^{(x-1)} z^{x}=\frac{f(z t)}{f(z)}
\end{aligned}
$$

dengan menyamakan (10) dengan nilai $E\left(X^{1}\right), \ldots, E\left(X^{k}\right)$ kemudian didiferensialkan terhadap $t$ didapatkan

$$
\begin{aligned}
& \left.G_{x}^{\prime}(t)\right|_{t=1}=E\left(X^{[1]}\right)=M^{[1]}=\frac{1}{f(z)} \sum_{x} a_{x} x z^{x}=\frac{z f^{\prime}(z)}{f(z)} \\
& \left.G_{x}^{\prime \prime}(t)\right|_{t=1}=E\left(X^{[2]}\right)=M^{[2]}=\frac{1}{f(z)} \sum_{x} a_{x} x(x-1) z^{x} \\
& =\frac{z^{2} f^{\prime \prime}(z)}{f(z)} \\
& \text {... } \\
& \left.G_{x}^{k}(t)\right|_{t=1}=E\left(X^{[k]}\right) \\
& =M^{[k]}=\frac{1}{f(z)} \sum_{x} a_{x} x(x-1) \ldots(x-(k+1)) z^{x} \\
& =\frac{z^{k} f^{k}(z)}{f(z)}
\end{aligned}
$$




\section{Hubungan Antara Momen Faktorial}

Hubungan antara momen faktorial dari distribusi deret pangkat diperoleh dengan menggunakan Teorema 3 dibawah ini.

\section{Teorema 5}

Jika $M^{[k]}$ momen faktorial ke- $k$ dan $M_{1}^{\prime}$ momen pertama, maka momen faktorial ke- $(k+1)$ yaitu :

$$
M^{[k+1]}=\frac{z d M^{[k+1]}}{d z}+M^{[k]} M_{1}^{\prime}-k M^{[k]},
$$

dengan $k$ bilangan bulat positif.

\section{Bukti}

Akan dibuktikan bahwa Teorema 5 benar.

$$
E\left(X^{(k)}\right)=M^{[k]}=\frac{1}{f(z)} \sum_{x=1}^{n} x^{[k]} a_{x} z^{x}
$$

selanjutnya didiferensialkan terhadap $z$, maka didapat :

$$
\begin{aligned}
& \frac{d M^{[k]}}{d z}=\frac{1}{f(z)} \sum_{x} x^{[k]} a_{x} x z^{(x-1)}-\frac{f^{\prime}(z)}{f^{2}(z)} \sum_{x} x^{[k]} a_{x} z^{x} \\
& =\frac{1}{z f(z)} \sum_{x} x^{[k+1]} a_{x} z^{x}-k \frac{1}{z f(z)} \sum_{x} x^{[k]} a_{x} z^{x} \\
& -\frac{f^{\prime}(z)}{f^{2}(z)} \sum_{x} x^{[k]} a_{x} z^{x} \\
& =\frac{1}{z} M^{[k+1]}+k \frac{1}{z} M^{[k]}-\frac{f^{\prime}(z)}{f(z)} M^{[k]} \\
& =\frac{1}{z} M^{[k+1]}+M^{[k]}\left(\frac{k}{z}-\frac{f^{\prime}(z)}{f(z)}\right) \\
& \frac{1}{Z} M^{[K+1]}=\frac{d M^{[k]}}{d z}-M^{[k]}\left(\frac{k}{z}-\frac{f^{\prime}(z)}{f(z)}\right) \\
& =z \frac{d M^{[k]}}{d z}-M^{[k]}\left(k-M_{1}^{\prime}\right) \\
& =z \frac{d M^{[k]}}{d z}-M^{[k]} k+M^{[k]} M_{1}^{\prime} \\
& M^{[k+1]}=z \frac{d M^{[k]}}{d z}-M^{[k]}\left(k-\frac{z f^{\prime}(z)}{f(z)}\right) \\
& =z \frac{d M^{[k]}}{d z}-M^{[k]}\left(k-M_{1}^{\prime}\right) \\
& =z \frac{d M^{[k]}}{d z}-M^{[k]} k+M^{[k]} M_{1}^{\prime}
\end{aligned}
$$

\section{Aplikasi Deret Pangkat Pada Beberapa Jenis} Distribusi Khusus

6.1 Distribusi Deret Negatif Logaritma

Jika diketahui deret sebagai berikut

$z+\frac{z^{2}}{z}+\frac{z^{3}}{z}+\ldots=\sum_{x=1} \frac{t^{x}}{x}=-\log (1-z) \quad|z|<1$

Didapat distribusi

$$
P(X=x)=-\frac{z^{x}}{x \log (1-z)}, 0<t<1, x=1,2, \ldots
$$

Maka diperoleh

$a_{x}=\frac{1}{x}, f(z)=-\log (1-t)=\log (1-z)^{-1}$

Berdasarkan definisi fungsi pembangkit momen didapatkan

$$
\begin{aligned}
M_{x}(t) & =\frac{e^{t}}{-\log (1-z)} \sum_{x} \frac{1}{x} e^{t(x-1)} z^{x} \\
& =-\frac{e^{t}}{\log (1-z)} \sum_{x} \frac{1}{x} e^{t(x-1)} z^{x} \\
& =\frac{\log \left(1-z e^{t}\right)}{\log (1-z)} \quad t<-\log (z)
\end{aligned}
$$

Selanjutnya diturunkan terhadap $t$ maka berdasarkan (11) didapat

$$
\left.\frac{M_{x}{ }^{\prime}(t)}{d t}\right|_{t=0}=E(X)=\frac{z f^{\prime}(z)}{f(z)}
$$

Karena $f(z)=-\log (1-z)$ maka

$$
f^{\prime}(z)=\frac{1}{(1-z)}
$$

Jadi

$$
\begin{aligned}
& \left.\frac{M_{x}{ }^{\prime}(t)}{d t}\right|_{t=0}=E(X)=\frac{z \frac{1}{(1-z)}}{-\log (1-z)} \\
& E(X)=-\frac{z}{(1-z) \log (1-z)} \\
& \left.\frac{M_{x}{ }^{\prime \prime}(t)}{d t^{2}}\right|_{t=0}=E\left(X^{2}\right)=\frac{z f^{\prime}(z)+z^{2} f^{\prime \prime}(z)}{f(z)}
\end{aligned}
$$

Karena $f^{\prime}(z)=\frac{1}{1-z}$ maka

$$
f^{\prime \prime}(z)=\frac{1}{(1-z)^{2}}
$$

Maka

$$
\begin{gathered}
\left.\frac{M_{x}{ }^{\prime \prime}(t)}{d t^{2}}\right|_{t=0}=E\left(X^{2}\right)=\frac{z\left(\frac{1}{(1-z)}\right)+z^{2}\left(\frac{1}{(1-z)}\right)}{-\log (1-z)} \\
=\frac{\frac{1}{(1-z)^{2}}\left((z(1-z))+z^{2}\right)}{-\log (1-z)} \\
E\left(X^{2}\right)=-\frac{z}{(1-z)^{2} \log ((1-z))}
\end{gathered}
$$

Jadi

$$
\begin{aligned}
\operatorname{Var}(X)= & E\left(x^{2}\right)-(E(x))^{2} \\
= & -\frac{z}{(1-z)^{2} \log (1-z)}-\left(-\frac{z}{(1-z) \log (1-z)}\right)^{2} \\
& =-\frac{z^{2}+z \log (1-z)}{(1-z)^{2} \log ^{2}(1-z)}
\end{aligned}
$$


Selanjutnya dari persamaan (10) diperoleh

$$
\begin{aligned}
G_{x}(t) & =\frac{t}{-\log (1-z)} \sum_{x} \frac{1}{x} t^{(x-1)} z^{x} \\
& =\frac{\log (1-z t)}{\log (1-z)}|t|<\frac{1}{z}
\end{aligned}
$$

Kemudian dari menyamakan persamaan (10) didapat

$$
\begin{aligned}
& \left.\frac{G_{x}^{\prime}(t)}{d t}\right|_{t=1}=E(X)=\mu_{1}=\frac{z f^{\prime}(x)}{f(z)}=\frac{z(1-z)^{-1}}{-\log (1-z)} \\
& E(X)=-\frac{z}{(1-z) \log (1-z)} \\
& \left.\frac{G_{x}^{\prime \prime}(t)}{d t^{2}}\right|_{t=1}=E\left(X^{2}\right)=\mu_{2}=\frac{z^{2} f^{\prime \prime}(z)}{f(z)}=\frac{z^{2}(1-z)^{2}}{-\log (1-z)} \\
& E\left(X^{2}\right)=-\frac{z^{2}}{(1-z)^{2} \log (1-z)}
\end{aligned}
$$

Selanjutnya moment faktorial ke- $k$

$$
\left.\frac{G_{x}{ }^{k}(t)}{d t^{k}}\right|_{t=1}=E\left(X^{(k)}\right)=-\frac{(k-1) ! z^{k}}{(1-z)^{k} \log (1-z)}
$$

\subsection{Distribusi Deret Log Pembagian}

\section{Diketahui dere}

$$
\begin{aligned}
2\left(z+\frac{z^{3}}{3}+\frac{z^{5}}{5}+\frac{z^{7}}{7}+\ldots\right) & =2 \sum_{x}\left(\frac{z^{2 x+1}}{2 x+1}\right) \\
& =\log \left(\frac{1+z}{1-z}\right), \quad|z|<1
\end{aligned}
$$

Didapatkan distribusi

$$
P(X=2 x+1)=\frac{2 z^{2 x+1}}{(2 x+1) \log \left(\frac{1+z}{1-z}\right)}
$$

Untuk $0<z<1, x=0,1,2$..

Didapat

$$
a_{x}=\frac{2}{2 x+1}, \quad f(z)=\log \left(\frac{1+z}{1-z}\right)
$$

Dari definisi fungsi pembangkit momen didapat

$$
\begin{aligned}
M_{x}(t) & =\frac{e^{t}}{\log \left(\frac{1+z}{1-z}\right)} \sum_{x} \frac{2}{2 x=1} e^{t(x-1)} z^{2 x+1} \\
& =\frac{2 e^{t}}{\log (1+z)} \sum_{x} \frac{e^{t(x-1)} z^{2 x+1}}{2 x+1} \\
& =\frac{\log \left(\frac{1+z e^{t}}{1-z e^{t}}\right)}{\log \left(\frac{1+z}{1-z}\right)}, t<-\log z
\end{aligned}
$$

Kemudian diturunkan terhadap $t$ diperoleh

$$
\left.\frac{M_{x}^{\prime}(t)}{d t}\right|_{t=0}=E(X)=\frac{z \frac{2}{\left(1-z^{2}\right)}}{\log \left(\frac{1+z}{1-z}\right)}
$$

Sebab

$$
\begin{aligned}
& f(z)=\log \left(\frac{1+z}{1-z}\right) \\
& f^{\prime}(z)=\frac{1}{\left(\frac{1+z}{1-z}\right)} \frac{2}{(1-z)^{2}}=\frac{2}{(1-z)^{2}} \\
&=\frac{2 t}{\left(1-z^{2}\right) \log \left(\frac{1+z}{1-z}\right)} \\
&\left.\frac{M_{x}^{\prime \prime}(t)}{d t^{2}}\right|_{t=0}=E\left(X^{2}\right)=\frac{2 f^{\prime}(z)+z^{2} f^{\prime \prime}(z)}{f(z)}
\end{aligned}
$$

Diketahui

$$
\begin{aligned}
& f^{\prime}(z)=2\left(1-z^{2}\right)^{-1} \\
& f^{\prime \prime}(z)=2(-1)\left(1-z^{2}\right)^{-2}(-2 z)=\frac{4 z}{\left(1-z^{2}\right)^{2}}
\end{aligned}
$$

Maka

$$
\begin{aligned}
E\left(X^{2}\right) & =\frac{z \frac{2}{\left(1-z^{2}\right)}+z^{2} \frac{4 z}{\left(1-z^{2}\right)^{2}}}{\log \left(\frac{1+z}{1-z}\right)} \\
& =\frac{\frac{1}{\left(1-z^{2}\right)^{2}}\left(2 z\left(1-z^{2}\right)+4 z^{3}\right)}{\log \left(\frac{1+z}{1-z}\right)} \\
& =\frac{\left(2 z-2 z^{3}+4 z^{3}\right)}{(1-2)^{2} \log \left(\frac{1+z}{1-z}\right)}
\end{aligned}
$$$$
E\left(X^{2}\right)=\frac{2\left(z^{3}+z\right)}{\left(1-z^{2}\right)^{2} \log \left(\frac{1+z}{1-z}\right)}
$$

Jadi

$$
\begin{aligned}
\operatorname{Var}(X) & =E(X)-(E(X))^{2} \\
& =\frac{2\left(z^{3}+z\right)}{\left(1-z^{2}\right)^{2} \log \left(\frac{1+z}{1-z}\right)}-\left(\frac{2 z}{(1-z) \log \left(\frac{1+z}{1-z}\right)}\right)^{2}
\end{aligned}
$$




$$
\operatorname{Var}(X)=2 z \frac{\left(1+z^{2}\right) \log \left(\frac{1+z}{1-z}\right)}{\left(1+z^{2}\right)^{2} \log \left(\frac{1+z}{1-z}\right)}
$$

Berdasarkan definisi fungsi pembangkit momen faktorial didapat

$$
\begin{aligned}
G_{x}(t) & =\frac{t}{\log \left(\frac{1+z}{1-z}\right)} \sum_{x} \frac{2}{(2 x+1)} t^{(x-1)} z^{(2 x+1)} \\
& =\frac{\log \left(\frac{1+z t}{1-z t}\right)}{\log \left(\frac{1+z}{1-z}\right)} \quad|t|<\frac{1}{2}
\end{aligned}
$$

Berdasarkan dengan menyamakan persamaan (10) didapat

$$
\begin{aligned}
& \left.\frac{G_{x}^{\prime}(t)}{d t}\right|_{t=1}=E\left(X^{(1)}\right)=\mu_{1}=\frac{z 2\left(1-z^{2}\right)^{-1}}{\log \left(\frac{1+z}{1-z}\right)} \\
& E\left(X^{(1)}\right)=\frac{2 z}{\left(1-z^{2}\right) \log \left(\frac{1+z}{1-z}\right)} \\
& \left.\frac{G_{x}^{\prime \prime}(t)}{d t^{2}}\right|_{t=1}=E\left(X^{2}\right)=\mu_{2}=\frac{z^{2}\left(\frac{4 z}{\left(1-z^{2}\right)^{2}}\right)}{\log \left(\frac{1+z}{1-z}\right)} \\
& E\left(X^{2}\right)=\frac{4 z^{3}}{\left(1-z^{2}\right)^{2} \log \left(\frac{1+z}{1-z}\right)} \\
& \left.\frac{G_{x}^{\prime \prime \prime}(t)}{d t^{2}}\right|_{t=1}=E\left(X^{3}\right)=\mu_{3}=\frac{z^{3} f^{\prime \prime \prime}(z)}{f(z)} \\
& =\frac{z^{3} \frac{4}{\left(1-z^{2}\right)^{3}}\left(1+3 z^{2}\right)}{\log \left(\frac{1+z}{1-z}\right)} \\
& =\frac{4 z^{3}\left(1+3 z^{2}\right)}{\left(1-z^{2}\right)^{3} \log \left(\frac{1+z}{1-z}\right)} \\
& E\left(X^{3}\right)=\frac{2 z^{2}\left(2 z+6 z^{3}\right)}{\left(1-z^{2}\right)^{3} \log \left(\frac{1+z}{1-z}\right)}
\end{aligned}
$$

\subsection{Distribusi Deret Invers Sinus (Arcsinus)}

Diketahui deret invers sinus

$z+\frac{1}{2} \frac{z^{3}}{3}+\frac{1}{2} \cdot \frac{3}{4} \cdot \frac{z^{5}}{5}+\ldots=z+\sum \frac{1 \cdot 3 \cdot 5(2 x-1) z^{2 x+1}}{2 \cdot 4 \cdot 5 \ldots(2 x) 2 x+1}$ $z+\frac{1}{2} \frac{z^{3}}{3}+\frac{1}{2} \cdot \frac{3}{4} \cdot \frac{z^{5}}{5}+\ldots=\sin ^{-1} z,|z|<1$

Dapat ditulis

$P(X=2 x+1)=\frac{1 \cdot 3 \ldots(2 x-1)}{2.4 \ldots(2 x)} \cdot \frac{z^{2 x+1}}{2 x+1}\left(\sin ^{-1} z\right)^{-1}$

untuk $0<z<1, \quad x=1,2, \ldots$

Didapat

$a_{x}=\frac{1 \cdot 3 \ldots(2 x-1)}{2 \cdot 4 \ldots(2 x)} \cdot \frac{1}{2 x+1}$

$f(z)=\sin ^{-1} z$

Dari definisi fungsi pembangkit momen didapat

$M_{x}(t)=\frac{e^{t}}{\sin ^{-1} z} \cdot \sum_{x} \frac{1 \cdot 3 \ldots(2 x-1)}{2 \cdot 4 \ldots .(2 x)} \cdot \frac{1}{2 x+1} \cdot e^{t(x-1)} z^{2 x+1}$ $=e^{t}\left(\sin ^{-1}\right)^{-1} \sum_{x} \frac{1 \cdot 3 \ldots(2 x-1)}{2 \cdot 4 \ldots(2 x)} \cdot \frac{1}{(2 x+1)} \cdot e^{t(x-1)} z^{2 x+1}$

$M_{x}(t)=\frac{\sin ^{-1}\left(z e^{t}\right)}{\sin ^{-1} z}, \quad t<-\log z$

Selanjutnya ditunjukan terhadap $t$. Maka berdasarkan hasil dari (43) didapatkan

$\left.\frac{M_{x}^{\prime}(t)}{d t}\right|_{t=0}=E(X)=\frac{z f^{\prime}(z)}{f(z)}$

karena

$f(z)=\sin ^{-1} z$

$f^{\prime}(z)=\frac{1}{\sqrt{1-z^{2}}}$

Maka

$$
\begin{aligned}
E(X)=\frac{z \frac{1}{\left(1-z^{2}\right)^{\frac{1}{2}}}}{\sin ^{-1} z} & =\frac{z}{\sqrt{1-z^{2}} \sin ^{-1} z} \\
\left.\frac{M_{x}^{\prime \prime}(t)}{d t^{2}}\right|_{t=0}=E\left(X^{2}\right) & =\frac{z \frac{1}{\sqrt{1-z^{2}}}+z^{2} \cdot \frac{z}{\left(1-z^{2}\right) \sqrt{1-z^{2}}}}{\sin ^{-1} z} \\
& =\frac{\frac{z}{\left(1-z^{2}\right) \sqrt{1-z^{2}}} \cdot\left(\left(1-z^{2}\right)+z^{2}\right)}{\sin ^{-1} z} \\
E\left(X^{2}\right) & =\frac{z}{\left(1-z^{2}\right) \sqrt{1-z^{2}} \sin ^{-1} z}
\end{aligned}
$$

Kemudian

$$
\begin{aligned}
\operatorname{Var}(X) & =E\left(X^{2}\right)-(E(X))^{2} \\
& =\frac{z}{\left(1-z^{2}\right) \sqrt{1-z^{2}} \sin ^{-1} z}-\left(\frac{z}{\sqrt{1-z^{2}} \sin ^{-1} z}\right)^{2} \\
& =\frac{z}{\left(1-z^{2}\right) \sqrt{1-z^{2}} \sin ^{-1} z}-\frac{z^{2}}{\left(1-z^{2}\right)\left(\sin ^{-1} z\right)^{2}}
\end{aligned}
$$




$$
\begin{gathered}
=\frac{z \sin ^{-1} z-z^{2} \sqrt{1-z^{2}}}{\left(1-z^{2}\right) \sqrt{1-z^{2}}\left(\sin ^{-1} z\right)^{2}} \\
\operatorname{Var}(X)=z \frac{\sin ^{-1} z-z \sqrt{1-z^{2}}}{\left(1-z^{2}\right) \sqrt{1-z^{2}}\left(\sin ^{-1} z\right)^{2}}
\end{gathered}
$$

Dengan menggunakan definisi fungsi pembangkit momen faktorial didapat

$$
\begin{aligned}
G_{x}(t) & =\frac{t}{\sin ^{-1} z} \sum_{x} \frac{1 \cdot 3 \ldots(2 x-1)}{2 \cdot 4 \ldots(2 x)}\left(\frac{1}{(2 x+1)}\right) t^{(x-1)} z^{(2 x+1)} \\
& =\frac{\sin ^{-1}(z t)}{\sin ^{-1} z},|t|<\frac{1}{z}
\end{aligned}
$$

Sehingga didapat

$$
\begin{aligned}
\left.G_{x}^{\prime}(t)\right|_{t=1}=E\left(X^{[1]}\right)=\mu_{1} & =\frac{z f^{\prime}(z)}{f(z)} \\
& =\frac{z\left(1-z^{2}\right)^{-\frac{1}{2}}}{\sin ^{-1} z} \\
& =\frac{z}{\sqrt{1-z^{2}} \sin ^{-1} z} \\
\left.G_{x}^{\prime \prime}(t)\right|_{t=1}=E\left(X^{[2]}\right)=\mu_{2} & =\frac{z^{2} f(z)}{f(z)} \\
& =\frac{z^{2} \frac{z}{\left(1-z^{2}\right) \sqrt{1-z^{2}}}}{\sin ^{-1} z} \\
& =\frac{z^{3}}{\left(1-z^{2}\right) \sqrt{1-z^{2}} \sin ^{-1} z} \\
\left.G_{x}^{\prime \prime \prime}(t)\right|_{t=1}=E\left(X^{[3]}\right)= & \frac{z^{3}+2 z^{5}}{\left(1-z^{2}\right)^{2} \sqrt{1-z^{2}} \sin ^{-1} z}
\end{aligned}
$$

$$
\operatorname{Var}(X)=2 z \frac{\left(1+z^{2}\right) \log \left(\frac{1+z}{1-z}\right)}{\left(1-z^{2}\right)^{2} \log \left(\frac{1+z}{1-z}\right)}
$$

3. Distribusi invers sinus (Arcsinus)

$$
\begin{aligned}
& E(X)=\frac{z}{\sqrt{1-z^{2}} \sin ^{-1} z} \\
& \operatorname{Var}(X)=z \frac{\sin ^{-1} z-z \sqrt{1-z^{2}}}{\left(1-z^{2}\right) \sqrt{1-z^{2}}\left(\sin ^{-1} z\right)^{2}}
\end{aligned}
$$

\section{DAFTAR PUSTAKA}

Bain, L. J; Engelhardt, M., (1991), "Introduction to Probability and Mathematical Statistics" The Duxbury Advanced Series In Statistics And Decision Sciences.

Dudewicz, E. J; Misra, S. N., (1995), "Statistika Matematika Modern", Penerbit ITB Bandung.

Kreyszig, E., (1993), Matematika Telaah Lanjutan (Statistik lanjutan) edisi ke-6. Penerbit PT Gramedia pustaka, Jakarta

Purcell, E. J; Varberg, D., (1992), "Kalkulus dan Geometri Analisis". Edisi Kelima, Penerbit Erlangga.

\section{KESIMPULAN}

Karakteristik parameter untuk model distribusi deret pangkat yang telah dibahas pada bab sebelumnya, ditarik kesimpulan tentang sifat-sifat analitik beberapa distribusi khusus sebagai berikut:

1. Distribusi deret negatif pembagian:

$$
\begin{aligned}
& E(X)=-\frac{z}{(1-z) \log (1-z)} \\
& \operatorname{Var}(X)=-\frac{z^{2}+z \log (1-z)}{(1-z)^{2} \log ^{2}(1-z)}
\end{aligned}
$$

2. Distribusi deret log pembagian:

$$
E(X)=\frac{z \frac{2}{\left(1-z^{2}\right)}}{\log \left(\frac{1+z}{1-z}\right)}
$$

